\title{
Biomasa, parámetros foliares y sintomatología en respuesta a diferentes niveles de manganeso, zinc y boro en curuba (Passiflora tripartita var. mollissima)
}

\section{Biomass, foliar parameters and symptomatology in response to different levels of manganese, zinc and boron in the banana passion fruit (Passiflora tripartita var. mollissima)}

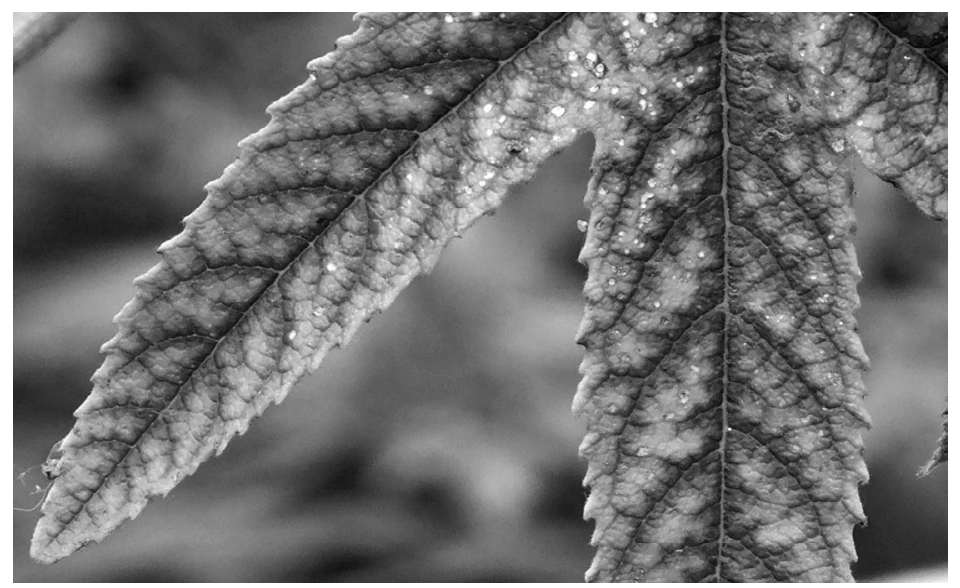

MIGUEL ÁNGEL LIZARAZO1, 3

CAMILO ANDRÉS HERNÁNDEZ1

GERHARD FISCHER ${ }^{1}$

MANUEL IVÁN GÓMEZ²

Clorosis intervenal causada por deficiencia de manganeso en hojas de curuba.

Foto: S. Hernández

\section{RESUMEN}

Para conocer el efecto del manganeso (Mn), zinc ( $\mathrm{Zn})$ y boro (B) sobre la distribución de masa seca (MS), crecimiento foliar y expresión de síntomas de deficiencia nutricional en curuba, se evaluaron tres niveles de fertilización: carencia (0), bajo (1) y elevado (2) para cada elemento, comparados con una fertilización completa (testigo), dispuestos en un diseño de bloques completamente al azar. Las plantas de curuba fueron trasplantadas en materas plásticas, con un sustrato de arena cuarcítica, realizando un riego y dos fertirriegos cada semana. Las variables evaluadas fueron masa seca total (MS), porcentaje de distribución de masa seca, área foliar (AF), número de hojas (NDH), área foliar específica (AFE) y relación de área foliar (RAF), además se realizó un registro fotográfico para reconocer la sintomatología de las deficiencias en hojas. Se encontró que en los diferentes niveles de cada elemento hubo restricción en la MS, siendo el nivel de carencia el más limitante. La distribución de masa seca demostró un aumento entre 1-14\% de acumulación en raíz para todos los tratamientos en comparación con el testigo. El NDH y AF fueron bastante limitados en el nivel de carencia de cada elemento, y en menor proporción los niveles 1 y 2. El AFE en todos los tratamientos exceptuando $\mathrm{Mn} 0$ y B0 fue mayor respecto al testigo. En la RAF todos los tratamientos, exceptuando Mn2 y Zn0, fueron similares al testigo. Signos visuales de deficiencia nutricional en hojas, sólo se observaron en los tratamientos $\mathrm{Mn} 0$, Zn0 y B0, evidenciado la sintomatología clásica para cada elemento, acorde a lo reportado en otras especies del mismo género.

Facultad de Agronomía, Departamento de Agronomía, Universidad Nacional de Colombia, Bogotá (Colombia).

INGEPLANT Ingeniería en nutrición de cultivos, Bogotá (Colombia).

Autor para correspondencia.malizarazot@unal.edu.co 


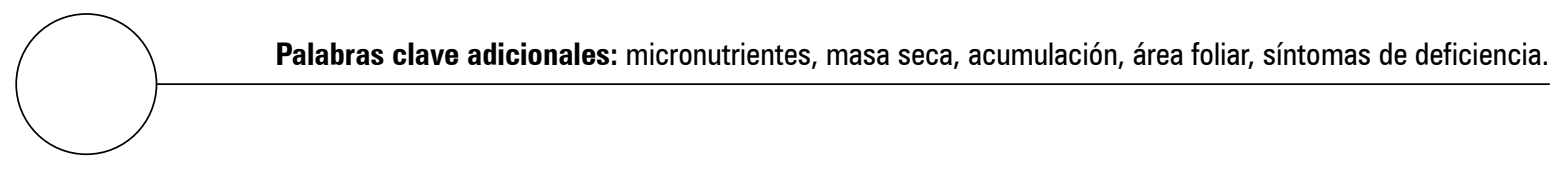

\section{ABSTRACT}

In order to observe the effects of manganese $(\mathrm{Mn})$, zinc $(\mathrm{Zn})$ and boron $(\mathrm{B})$ on dry mass $(\mathrm{DM})$ allocation, canopy growth and nutritional deficiency symptom expression in the banana passion fruit; three levels of fertilization were evaluated: none (0), low (1) and high (2), for each element and compared to complete fertilization (control), with a randomized complete block design. The banana passion fruit plants were transplanted to plastic pots containing a substrate made of quartz sand, with irrigation twice a week and fertigation once a week. The evaluated variables were total dry weight (TDW), percentage of dry mass allocation, leaf area (LA), number of leaves (NL), specific leaf area (SLA) and leaf area ratio (LAR). In addition, a photographic record was taken to recognize symptomatology in the leaves. At different levels of each element, restriction of the TDW was found, with the zero level being the most limiting. The DM distribution showed an increase between 1 and 14\% of accumulation in roots of the treatments compared to the control. The NL and LA were quite limited at the zero level of each element and, to a lesser extent, in the levels 1 and 2. The SLA was high in all treatments, except $\mathrm{MnO}$ and $\mathrm{B} 0$, relative to the control. The LAR was equal to the control in all treatments, except $\mathrm{Mn} 2$ and $\mathrm{Zn} 0$. Visual signs of nutritional deficiency in the leaves were observed only in the $\mathrm{Mn} 0, \mathrm{Zn} 0$ and $\mathrm{B} 0$ treatments, evidencing classic symptoms for each element, according to reports on other species of the same genus.

Additional key words: micronutrients, dry mass, accumulation, leaf area, deficiency symptoms.

Fecha de recepción: 25-01-2013

Aprobado para publicación: 28-05-2013
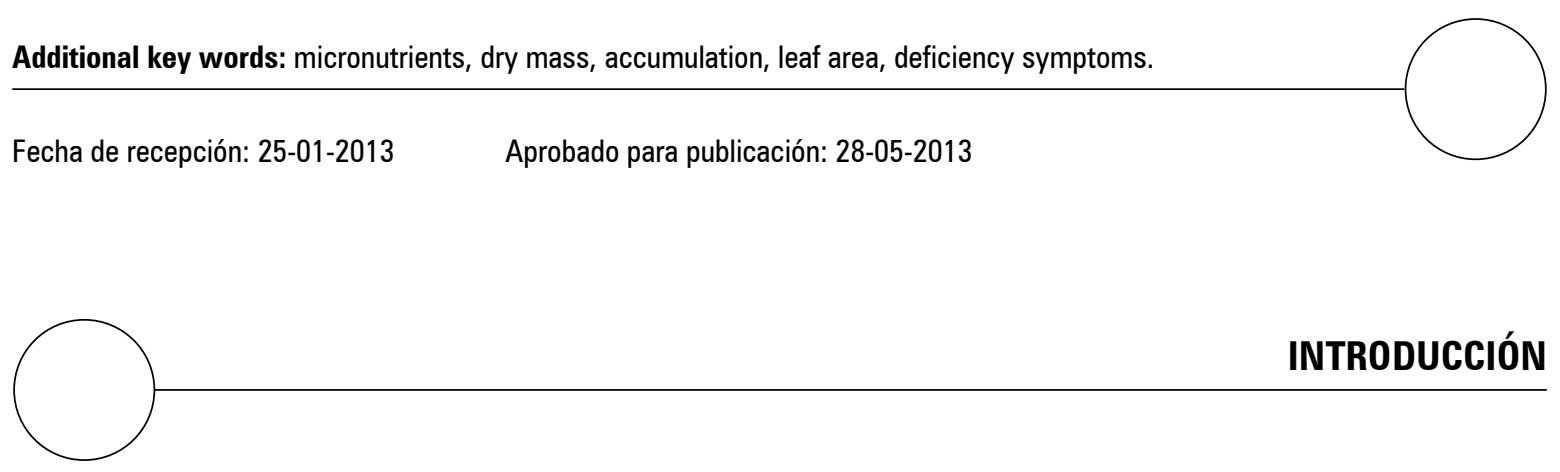

La curuba, parcha o tumbo, es una planta originaria de los valles andinos. En Colombia existe un amplio banco genético con cerca de 21 especies, siendo la curuba de castilla (Passiflora tripartita var. mollissima) una de las más cultivadas a nivel comercial o de huerto casero, pues en departamentos como Boyacá, Cundinamarca y Nariño se ha aprovechado la mayor área comercial de este cultivo, generando ingresos económicos a pequeños y medianos agricultores (Lizarazo et al., 2013). A pesar de esto, no existe un avance sólido en investigación para la especie, y en gran medida su cultivo se desarrolla de forma empírica (Primot et al., 2005; Campos y Quintero, 2012).

En general, los sistemas de producción de curuba en el país se encuentran en clima frío a frío moderado y se caracterizan por tener suelos poco profundos, de horizontes endurecidos y de baja a mediana fertilidad (Miranda y Carranza, 2010), por lo que las plantas están expuestas a diferentes condiciones en la disponibilidad de nutrientes, que pueden influenciar procesos bioquímicos, generando alteraciones fisiológicas y morfológicas hasta el punto de reducir la canti- 
dad y calidad de la cosecha, como respuesta a dicho entorno (McCarthy y Enquist, 2007). Pero las limitaciones de tipo nutricional, como en el caso de los microelementos, no suelen ser lo bastante severas para producir síntomas visuales, por lo que son difíciles de detectar y reconocer (Thompson y Troeh, 1988).

En este sentido, a pesar de que las necesidades nutricionales de la curuba no han sido bien determinadas, algunos datos evidenciados en campo y experiencias aisladas de ciertos autores pueden ayudar a los productores en el manejo de la fertilización del cultivo de curuba (Campos, 2001; Campos y Quintero, 2012). Teniendo en cuenta que dicho manejo, a través de fertilizantes y enmiendas puede representar entre $25-30 \%$ de los costos de producción, es importante asegurar que la planta disponga de las cantidades adecuadas de los elementos esenciales para expresar su potencial genético, a través del acercamiento a una fertilización más ajustada a las necesidades de la especie, siendo de gran importancia las herramientas de diagnóstico, interpretación y recomendación del estado nutricional del cultivo (Gómez, 2012).

Por lo tanto, el estudio tuvo como objetivo establecer el efecto de diferentes dosis de fertilización de manganeso, zinc y boro, sobre la biomasa, parámetros foliares y síntomas de deficiencia en plantas de curuba.

\section{MATERIALES Y MÉTODOS}

El ensayo se desarrolló bajo condiciones de invernadero en la Facultad de Agronomía de la Universidad Nacional de Colombia, Bogotá, con temperatura promedio interna de $20^{\circ} \mathrm{C}$ y humedad relativa de $80 \%$. Plantas de curuba de Castilla (Passiflora tripartita var. mollissima), obtenidas de un vivero comercial, fueron podadas $35 \mathrm{~d}$ después de emergencia para estimular la brotación de yemas laterales y elegir un tallo principal de altura uniforme. Durante el experimento las plantas permanecieron en macetas plásticas de $5 \mathrm{~kg}$, con un sustrato inerte de arena cuarcítica (calibre $\leq 1 \mathrm{~mm}$ ) y cuarzo $(\leq 4 \mathrm{~mm})$, en proporción 1:1. La distribución espacial fue de $0,5 \mathrm{~m}$ entre plantas y 1,0 m entre hileras.

Se utilizó un sistema de tutorado en espaldera vertical, con dos líneas de alambre a 1 y $2 \mathrm{~m}$ de altura. El meristemo apical fue podado cuando las plantas alcanzaron la línea de alambre superior, estimulando así la brotación de tallos secundarios, de los cuales se seleccionaron dos por cada línea de alambre. Periódicamente se retiraron los brotes laterales nuevos de la rama principal para favorecer el crecimiento de los tallos secundarios.

Los tratamientos estuvieron compuestos por tres niveles o dosis de fertilización para cada uno de los elementos Mn, Zn y B, siendo (0): carencia total del elemento; (1): cada elemento se encontraba en menor proporción que la dosis comercial o testigo y (2): cada elemento estaba en mayor proporción que la dosis comercial, para un total de $10(3 \times 3+1)$ tratamientos. Para la dosificación de la cantidad de cada nutriente se usó como herramienta guía, el programa de fertilización de un cultivo comercial en etapa de desarrollo vegetativo, recomendado por M.I. Gómez (comunicación personal, 2010) (tabla 1).

Las fuentes usadas en las soluciones madre fueron: urea $\left(\mathrm{CH}_{4} \mathrm{~N}_{2} \mathrm{O}\right)$, fosfato monoamónico $\left(\mathrm{NH}_{4} \mathrm{H}\right.$ $\left.{ }_{2} \mathrm{PO}_{4}\right)$, fosfato monopotásico $\left(\mathrm{KH}_{2} \mathrm{PO}_{4}\right)$, cloruro de potasio $(\mathrm{KCl})$, nitrato de potasio $\left(\mathrm{KNO}_{3}\right)$, sulfato de potasio $\left(\mathrm{K}_{2} \mathrm{SO}_{4}\right)$, ácido fosfórico $\left(\mathrm{H}_{3} \mathrm{PO}_{4}\right)$, sulfato de magnesio Heptah $\left(\mathrm{MgSO}_{4} \cdot 7 \mathrm{H}_{2} \mathrm{O}\right)$, nitrato de magnesio $\left(\mathrm{Mg}\left(\mathrm{NO}_{3}\right)_{2} \cdot 6 \mathrm{H}_{2} \mathrm{O}\right)$, octaborato de sodio $\left(\mathrm{Na}_{2} \mathrm{~B}_{8} \mathrm{O}_{13} \cdot 4 \mathrm{H}_{2} \mathrm{O}\right)$, sulfato de zinc $\left(\mathrm{ZnSO}_{4}\right)$, sulfato de hierro $\left(\mathrm{FeSO}_{4}\right)$, sulfato de cobre técnico $\left(\mathrm{CuSO}_{4} \cdot 5 \mathrm{H}_{2} \mathrm{O}\right)$, nitrato de calcio $\left(\mathrm{Ca}\left(\mathrm{NO}_{3}\right)_{2}\right)$, cloruro de calcio $\left(\mathrm{CaCl}_{2}\right)$, sulfato de manganeso $\left(\mathrm{MgSO}_{4} \cdot 7 \mathrm{H}_{2} \mathrm{O}\right)$, molibdato de sodio $\left(\mathrm{Na}_{2} \mathrm{MoO}_{4}\right)$, sulfato de cobalto $\left(\mathrm{CoSO}_{4}\right)$, ácido 
Tabla 1. Concentración de elementos minerales (mg L-1) en la solución madre (tomado de Lizarazo et al., 2013).

\begin{tabular}{|l|r|r|r|r|r|r|r|r|r|r|r|r|r|r|}
\hline $\begin{array}{l}\text { Tratamiento } \\
\begin{array}{l}\text { Testigo } \\
\text { comercial }\end{array}\end{array}$ & 221,42 & 62,21 & 235,52 & 144,16 & 34,59 & 42,03 & 0,63 & 0,42 & 0,13 & 1,54 & 1,00 & 0,06 & 0,01 \\
\hline Mn0 & 221,42 & 62,21 & 235,52 & 144,16 & 34,59 & 42,03 & 0,63 & 0,42 & 0,13 & 0 & 1,00 & 0,06 & 0,01 \\
\hline Mn1 & 221,42 & 62,21 & 235,52 & 144,16 & 34,59 & 42,03 & 0,63 & 0,42 & 0,13 & 0,39 & 1,00 & 0,06 & 0,01 \\
\hline Mn2 & 221,42 & 62,21 & 235,52 & 144,16 & 34,59 & 42,03 & 0,63 & 0,42 & 0,13 & 2,32 & 1,00 & 0,06 & 0,01 \\
\hline Zn0 & 221,42 & 62,21 & 235,52 & 144,16 & 34,59 & 42,03 & 0 & 0,42 & 0,13 & 1,54 & 1,00 & 0,06 & 0,01 \\
\hline Zn1 & 221,42 & 62,21 & 235,52 & 144,16 & 34,59 & 42,03 & 0,32 & 0,42 & 0,13 & 1,54 & 1,00 & 0,06 & 0,01 \\
\hline Zn2 & 221,42 & 62,21 & 235,52 & 144,16 & 34,59 & 42,03 & 0,95 & 0,42 & 0,13 & 1,54 & 1,00 & 0,06 & 0,01 \\
\hline B0 & 221,42 & 62,21 & 235,52 & 144,16 & 34,59 & 42,03 & 0,63 & 0 & 0,13 & 1,54 & 1,00 & 0,06 & 0,01 \\
\hline B1 & 221,42 & 62,21 & 235,52 & 144,16 & 34,59 & 42,03 & 0,63 & 0,21 & 0,13 & 1,54 & 1,00 & 0,06 & 0,01 \\
\hline B2 & 221,42 & 62,21 & 235,52 & 144,16 & 34,59 & 42,03 & 0,63 & 0,63 & 0,13 & 1,54 & 1,00 & 0,06 & 0,01 \\
\hline
\end{tabular}

cítrico anhidro (acidificante) $\left(\mathrm{C}_{6} \mathrm{H}_{8} \mathrm{O}_{7}\right)$ y EDTA sal sódica $\left(\mathrm{C}_{10} \mathrm{H}_{14} \mathrm{~N}_{2} \mathrm{Na}_{2} \mathrm{O}_{8} \cdot 2 \mathrm{H}_{2} \mathrm{O}\right)$.

La evaluación correspondió a un único muestreo destructivo al noveno mes después de trasplante, finalizando el ciclo vegetativo de la planta; etapa en la que las ramas principales estuvieron formadas e inició la emisión de brotes florales. La aplicación de los tratamientos se inició $68 \mathrm{~d}$ después de trasplante. Semanalmente se realizó un riego y dos fertirriegos, con un volumen inicial en cada aplicación de $300 \mathrm{~mL}$ por planta, que aumentó a $600 \mathrm{~mL}$ al final del ciclo.

El número de hojas (NDH), área foliar (AF), área foliar específica (AFE) y relación de área foliar (RAF) fueron evaluadas para comprender la relación que existe entre los diferentes tratamientos con el área fotosintética y la consecuente producción de biomasa. La cuantificación de estas fue realizada en el laboratorio de Fisiología de Cultivos de la Facultad de Agronomía, Universidad Nacional de Colombia, Bogotá. El NDH se estableció a través de un conteo de hojas maduras o completamente expandidas. Para la determinación de la masa seca (MS), fueron puestos hojas, tallos y raíz por separado, en una estufa de secado a $70^{\circ} \mathrm{C}$ por $48 \mathrm{~h}$, para su posterior pesado en una balanza analítica. El AF se determinó con un planímetro Licor LI-3100.
Con la información obtenida en las mediciones directas, respecto a MS y AF, se calcularon los índices de crecimiento RAF y AFE, utilizando las siguientes fórmulas (Hunt, 1990):

Área foliar específica (AFE): relación entre el área foliar total (AF) y la masa seca total de las hojas (MSH):

$$
A F E=\frac{A F}{M S H}\left(\mathrm{~cm}^{2} \mathrm{~g}^{-1}\right)
$$

Relación de área foliar (RAF): relación entre el área foliar total (AF) y la masa seca total (MST) de la planta:

$$
R A F=\frac{A F}{M S T}\left(\mathrm{~cm}^{2} \mathrm{~g}^{-1}\right)
$$

Adicionalmente se realizó una caracterización visual y registro fotográfico de los síntomas presentados en hojas bajo tratamientos de carencia.

El experimento se realizó bajo un diseño de bloques completos al azar (BCA) con cuatro repeticiones en donde la unidad experimental fue una planta. Para el análisis estadístico de los datos, se utilizó el programa SAS ${ }^{\circledR}$ versión 9.1, en el que 
se determinó el análisis de varianza (Anova) y se realizó la prueba de rango múltiple de Duncan para establecer la diferencia estadística entre promedios.

\section{RESULTADOS Y DISCUSIÓN}

\section{Acumulación de masa seca}

\section{Manganeso}

El Mn interviene en la síntesis de hormonas, proteínas y aceites, en el metabolismo y asimilación de nitrógeno, está presente en la activación metabólica de diferentes enzimas y forma metaloproteínas que a su vez son componentes de dos enzimas, una de actividad antioxidante como la Mn-superóxido dismutasa (MnSOD) importante en la protección contra los radicales libres de oxigeno $\mathrm{O}_{2}^{-}$, y la otra participa con el cloro en la disociación fotosintética del agua (Humphries et al., 2007; Kirkby y Römheld, 2008; Broadley et al., 2012).

El nivel 0 de $\mathrm{Mn}$ presentó la menor MS respecto a los demás tratamientos (figura 1), posiblemente por el deterioro de los procesos fisiológicos descritos anteriormente. Singh et al. (2001) reportan resultados similares en diferentes genotipos de menta (Mentha spicata) bajo carencia de $\mathrm{Mn}$, encontrando limitación en la MS acumulada, reducción importante en la fotosíntesis neta y el contenido de clorofilas.

Los niveles 1 y 2 de este elemento presentaron valores significativamente menores $(P \leq 0,05)$ de acumulación de MS en comparación con el testigo, resultados similares a los reportados en girasol (Helianthus annuus), tabaco (Nicotiana tabacum) y triticale (x Triticosecale) (László, 2008).

Una pequeña cantidad de $\mathrm{Mn}$ es esencial en las plantas pero su abundancia puede resultar perjudicial, existiendo un nivel crítico de toxicidad particular para cada especie (Thompson y
Troeh, 1988). En trigo (Triticum aestivum cv. Stacy), concentraciones deficientes como elevadas de $\mathrm{Mn}$ pueden afectar la síntesis de isoprenoides, que está relacionada con la producción de carotenoides, clorofila, ácido giberélico y esteroles, esenciales para la fotosíntesis y crecimiento (Wilkinson y Ohki, 1988; Singh et al., 2001).

Por su parte Kitao et al. (1999) identificaron en plántulas de especies leñosas, que una elevada concentración de manganeso afecta la acumulación de MS y la tasa relativa de crecimiento, por disminución en la tasa de fotosíntesis neta debida a una elevada acumulación de Mn en hojas, que se relaciona con una reducción en el contenido de clorofilas (González y Lynch, 1997), fenómeno que posiblemente causó la respuesta del tratamiento con dosis por encima de la comercial. En este sentido, la fitotoxicidad de manganeso puede manifestarse en una reducción en la biomasa, la fotosíntesis, y desordenes bioquímicos como estrés oxidativo (Millaleo et al., 2010).

\section{Zinc}

En plantas superiores hay enzimas que contienen zinc, como la ARN polimerasa y la anhidrasa carbónica (AC). Cuando hay escasez de este elemento la actividad de la AC disminuye, mostrando una baja tasa de fotosíntesis, pues esta enzima presente en el estroma de los cloroplastos facilita la transferencia de $\mathrm{CO}_{2} / \mathrm{HCO}_{3}$ para la fijación fotosintética del $\mathrm{CO}_{2}$. Además cuando se suprime el zinc hay inhibición en la síntesis de proteínas, debido a la disminución de la ARN polimerasa, causando disminución en el crecimiento (Kirkby y Römheld, 2008; Broadley et al., 2012). Probablemente la carencia de zinc desencadenó estos procesos, lo que ocasionó la baja acumulación de MS observada en Zn0 (figura 1).

Los tratamientos $\mathrm{Zn} 1$ y $\mathrm{Zn} 2$ fueron estadísticamente mayores a $Z n 0$ y menores que el testigo $(P \leq 0,05)$. La baja MS acumulada en $Z n 2$ puede ser indicativo de un nivel excesivo, concordando con lo reportado en fresa (Fragaria sp. cv. 
Camarosa) (Casierra-Posada y Poveda, 2005) y brócoli (Brassica oleracea var. Itálica) (CasierraPosada et al., 2010), en las que el exceso de zinc disminuyó la MS, como consecuencia de la irregularidad en el funcionamiento del aparato fotosintético y reducción en el contenido de pigmentos fotosintéticos.

\section{Boro}

La MS en los diferentes niveles de boro presentó diferencias significativas entre sí $(P \leq 0,05)$ y fue similar a la observada en los elementos anteriores (figura 1). En el nivel de carencia (B0), la poca MS acumulada pudo ser consecuencia de un desequilibrio en las funciones que este cumple en la planta, puesto que es elemento estructural de la membrana celular, influye en la elongación de la raíz e interviene en procesos metabólicos como la síntesis de ácidos nucleicos, proteínas, aminoácidos, almidón, auxinas y fenoles (Gupta, 2007), participa en el transporte de azúcares y lignificación de la pared celular (Loomis y Durst, 1992) y su ausencia tiene un efecto negativo en la división y elongación celular (Lukaszewski y Blevins, 1996). Siendo posible que los tejidos meristemáticos cesen su crecimiento afectando el desarrollo de nuevos órganos y por consiguiente la acumulación de biomasa como en lo reportado por Da Silva et al. (2008) en Ricinus communis.

La MS en los tratamientos B1 y B2 fue menor comparado al testigo $(P \leq 0,05)$, sugiriendo que este parámetro es influenciado, hasta cierto punto, por la cantidad de boro suministrado, pues dosis menores al igual que mayores, respecto a la recomendada (testigo), restringen la ganancia de MS. Análogamente Herrera et al. (2010) reportaron que cantidades elevadas de boro disminuyen el crecimiento de órganos vegetativos, el contenido de clorofila y la tasa fotosintética, debido a desbalances osmóticos e incapacidad de resistencia al daño fotoxidativo. Este resultado es contrastante con lo reportado en canola (Brassica napus) y girasol (Helianthus annuus) en los que a mayor cantidad de boro suministrado, las plantas acumulan mayor MS (Asad et al., 2002).

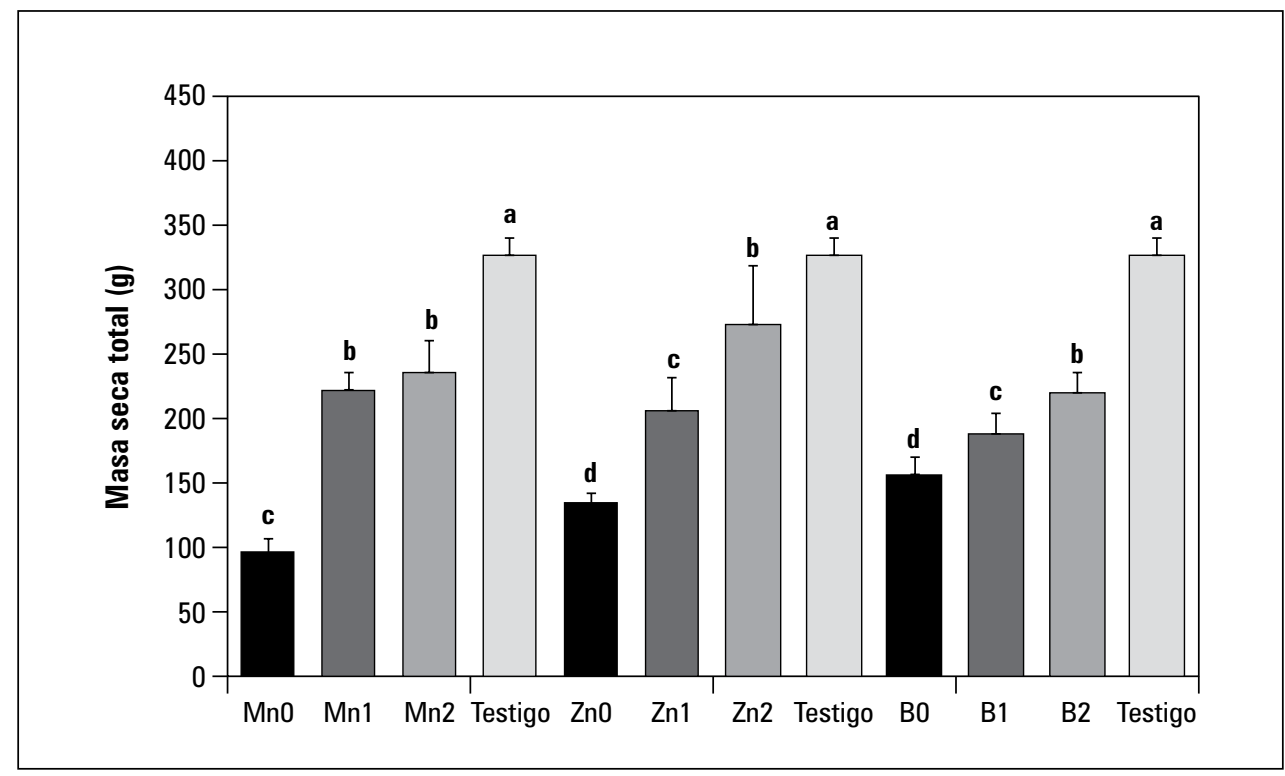

Figura 1. Efecto de diferentes niveles de $\mathrm{Mn}, \mathrm{Zn}$ y B en la masa seca total de plantas de curuba (Passiflora tripartita var. mollissima). Promedios con letras distintas, entre niveles de un mismo elemento, indican diferencia significativa según la prueba de Duncan $(P \leq 0,05)$. Las barras sobre las columnas indican desviación estándar. 


\section{Distribución de masa seca}

\section{Manganeso}

El tratamiento $\mathrm{Mn} 0$, acumuló 59,6\% de MS en raíz, resultado que contrasta con lo planteado por Broadley et al. (2012), pues la carencia de manganeso inhibe el crecimiento de las raíces, efecto tanto de una baja acumulación de carbohidratos, como reducida elongación celular. Paralelamente los tratamientos Mn1 y Mn2 tuvieron un esquema similar con 57,1 y $61,2 \%$, respectivamente (figura 2).

Diversos estudios demuestran que el efecto del manganeso en la distribución de la MS no tiene un patrón generalizado, indicando que puede variar según la especie. Por ejemplo, en espinaca (Spinacia oleraceae) no hubo alteración en la distribución de MS a mayor concentración de Mn (Casierra-Posada et al., 2012), mientras que en Populus cathayana un nivel elevado de manganeso redujo significativamente la parte aérea (Lei et al., 2007). En vid (Vitis vinifera) una alta concentración limitaba la MS en particular de la raíz, reduciendo la relación raíz:parte aérea, además causó una reducción importante de los pelos radicales (Mou et al., 2011) y en trigo (Triticum aestivum) niveles deficientes como excesivos restringen el número de tallos y el peso seco en raíz y parte aérea, originando también una baja proporción raíz:parte aérea (Ohki, 1984).

\section{Zinc}

Los tres niveles de zinc presentaron una tendencia similar, destinando a raíz entre 9,5 y $13,9 \%$ más MS que el testigo, sugiriendo que a pesar de haber diferencias significativas $(P \leq 0,05)$ en la MS acumulada por órgano vegetativo (tabla 2 ), no hay cambios marcados en los patrones de distribución en la planta (figura 2). Sin embargo, en cuanto a la carencia de este elemento, en cultivares de trigo duro (Triticum durum) y blando (Triticum aestivum) existe una alta relación raíz:parte aérea, resultado de un daño oxidativo en la parte aérea que limita su acumulación de MS (Cakmak et al., 1996). Marschner et al. (1996) complementan que dicha deficiencia combinada con una elevada intensidad lumínica conlleva a una foto-oxidación de los pigmentos del cloroplasto deteriorando el crecimiento y funcionamiento de las hojas fuente y disminuyendo la exportación de fotosintatos, debido al aumento del nivel de $\mathrm{O}_{2}{ }^{-}$en las células por deterioro en la actividad antioxidante de la $\mathrm{Cu} / \mathrm{Zn}$ superóxido dismutasa (SOD).

En brócoli (Brassica oleracea var. Itálica) la distribución de MS entre órganos de la parte aérea no fue afectada por dosis elevadas de zinc, mientras que en raíz hubo diferencias respecto al control, lo que representa una baja relación raíz:parte aérea (Casierra-Posada et al., 2010).

\section{Boro}

La tendencia de partición de MS para los niveles 1 y 2 de boro fue similar, mientras que B0 acumuló $12,7 \%$ más MS en raíz, comparados con el testigo (figura 2), en contraste con el síntoma típico en el que la carencia de este elemento inhibe el crecimiento radical, pues se afecta negativamente la diferenciación y crecimiento celular en raíces y pelos radicales, probablemente por un desequilibrio en el metabolismo y concentración de ascorbato en las raíces y otros desordenes a nivel de la membrana celular (Lukaszewski y Blevins, 1996; Goldbach et al., 2001; Broadley et al., 2012).

Es sabido que las plantas tienden a presentar mayor biomasa en raíz para ampliar la zona de exploración radical bajo condiciones limitadas de agua o nutrientes (McCarthy y Enquist, 2007), pero Wilson (1988) tras una extensa revisión, plantea que los mecanismos que determinan la partición de dicha MS en la planta y la relación raíz:parte aérea con relación a ciertos modelos alométricos, no responden a deficiencias de elementos menores y en caso de haber efecto, es bastante reducido, lo cual parece aclarar en cierta medida los re- 
sultados obtenidos en esta variable, en el que se encontraron diferencias estadísticas $(P \leq 0,05)$ en la $\mathrm{MS}$ acumulada en órganos vegetativos de los tratamientos de un mismo elemento, como en el caso del manganeso y zinc (tabla 2). Asimismo, la distribución de fotoasimilados y otros parámetros biométricos son controlados principalmente por elementos como el nitrógeno, fósforo o potasio (Hirose, 1987; Santiago et al., 2012).

En girasoles (Helianthus annuus) la cantidad de boro aplicado influyó en la distribución de MS, posiblemente por un mejor transporte de carbohidratos y hormonas, dado que es una de las funciones de este elemento (Goldbach et al., 2001; Zahoor et al., 2011). En curuba, bajo deficiencia de elementos mayores en etapa vegetativa, el patrón de distribución de MS presentó un alto componente en el sistema radical, mayor al 50\% de la MS, argumentando que esta especie tiene la tendencia de almacenar mayor MS en raíces, para luego retranslocarla a órganos como flores, frutos y meristemos, cuando sea necesario, por ejemplo al momento de realizar la poda de producción (Cabezas y Sánchez, 2008).

\section{Parámetros foliares}

\section{Manganeso}

La carencia de Mn afectó los valores de NDH y AF, siendo dos a tres veces menores comparados con los demás niveles del elemento y testigo (figura 3). Tanto el AFE como la RAF fueron estadísticamente similares al testigo $(P \leq 0,05)$, lo que indica una respuesta parecida de acumulación de MS en hojas y producción neta de biomasa con relación al área foliar, que cuando hay suficiencia del elemento. La reducción del AF bajo carencia de Mn es acorde con lo reportado en soya por Weiland et al. (1975).

E1 NDH y AF en Mn1 no fueron tan limitados como en $\mathrm{Mn} 0$, pero significativamente menores que $\mathrm{Mn} 2$ y el testigo $(P \leq 0,05)$. El AFE fue similar a Mn2 y a su vez mayor que el testigo, mientras que la RAF no fue diferente de los demás tratamientos. Esto muestra que hojas más pequeñas y delgadas permitieron similar ganancia neta de asimilados por unidad de área foliar que Mn0, Mn2 y el testigo, sin embargo la MS

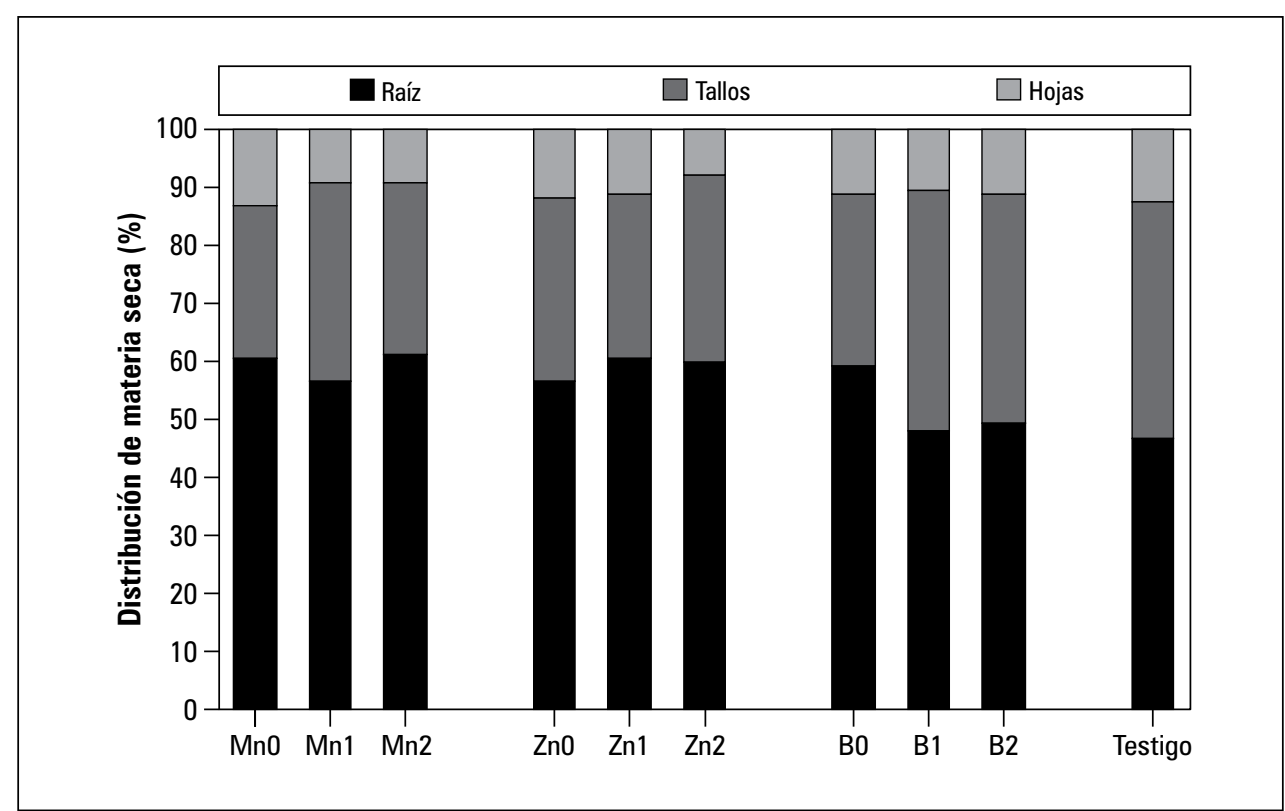

Figura 2. Efecto de diferentes niveles de $\mathrm{Mn}, \mathrm{Zn}$ y B en la distribución de masa seca en plantas de curuba (Passiflora tripartita var. mollissima). 


\begin{tabular}{|c|c|c|c|c|c|c|c|c|c|c|}
\hline \multirow{2}{*}{ Órgano } & \multicolumn{10}{|c|}{ Tratamiento } \\
\hline & Testigo & $\mathrm{MnO}$ & Mn1 & Mn2 & $\mathrm{ZnO}$ & $\mathrm{Zn} 1$ & $\mathrm{Zn2}$ & BO & B1 & B2 \\
\hline Hojas & 39,03 a & $12,04 \mathrm{c}$ & $20,10 \mathrm{~b}$ & $20,98 b$ & $15,30 \mathrm{c}$ & $22,91 \mathrm{~b}$ & $20,91 \mathrm{bc}$ & $17,10 \mathrm{c}$ & $19,64 \mathrm{bc}$ & $23,20 \mathrm{~b}$ \\
\hline Tallos & 133,33 a & $25,34 \mathrm{c}$ & $74,44 \mathrm{~b}$ & $69,97 \mathrm{~b}$ & $42,33 \mathrm{~d}$ & $57,00 \mathrm{c}$ & $87,56 \mathrm{~b}$ & $45,40 \mathrm{c}$ & $77,11 \mathrm{~b}$ & $87,12 b$ \\
\hline Raíz & 152,48 a & $57,77 \mathrm{c}$ & $126,00 \mathrm{~b}$ & $143,47 \mathrm{ab}$ & $74,90 \mathrm{c}$ & $124,55 b$ & 163,54 a & $92,37 \mathrm{bc}$ & $89,60 \mathrm{c}$ & $107,22 b$ \\
\hline Total & $324,83 a$ & $95,15 \mathrm{c}$ & $220,54 \mathrm{~b}$ & $234,42 b$ & $132,54 \mathrm{~d}$ & $204,45 \mathrm{c}$ & $272,00 \mathrm{~b}$ & $154,86 \mathrm{~d}$ & $186,34 \mathrm{c}$ & $217,54 b$ \\
\hline
\end{tabular}

estuvo limitada, acorde a ensayos de nutrición con Mn, en plantas jóvenes de abedul (Betula pendula), en donde valores altos de AFE estuvieron asociados a una baja tasa de crecimiento (Göransson, 1994).

En Mn2 el NDH y AF fueron estadísticamente similares al testigo $(P \leq 0,05)$, contrastando con lo reportado en Populus cathayana por Lei et al. (2007), donde el AF se redujo por exceso de Mn, debido al estrés oxidativo causado por acumulación de peróxido de hidrógeno $\left(\mathrm{H}_{2} \mathrm{O}_{2}\right)$ y malondialdehído (MDA). Los parámetros AFE y la RAF fueron mayores que el testigo, sugiriendo láminas foliares delgadas de mayor área pero probablemente poco eficientes en la producción de biomasa, lo cual se podría comprobar con la medición de otros parámetros como la tasa de asimilación neta (Cramer y Nowak, 1992).

En diferentes especies de abedul no hubo variación en la RAF bajo diferentes niveles de Mn, sin embargo, su exceso redujo la tasa relativa de crecimiento, fenómeno que se adujo a la acumulación de $\mathrm{Mn}$ en hojas, que redujo la actividad fotosintética y en consecuencia el crecimiento (Kitao et al., 1999).

\section{Zinc}

En cuanto al NDH no hubo diferencias significativas entre los niveles de zinc, siendo menores que el testigo $(P \leq 0,05)$. Zn0 presentó la menor AF sin ser estadísticamente diferente a Zn1 $(P \leq 0,05)$. La deficiencia de este elemento reduce la concentración ácido indolacético (AIA), ya sea por la limitación en su biosíntesis, pues actúa en la formación de triptófano, precursor del AIA, o por degradación oxidativa de este compuesto. Eventualmente el bajo nivel de esta hormona junto con el de las giberelinas y otros factores asociados a dicha carencia, pueden ser la causa del truncamiento del AF confirmando el fenómeno de "hojas pequeñas" (figura 3) (Broaley et al., 2012).

Feng et al. (2012) encontraron en cardamomo (Amomum villosum) que deficiencias de zinc, manganeso y cobre generan estrés oxidativo, aceleran la senescencia foliar y limitan el crecimiento, lo que puede relacionarse con el bajo $\mathrm{NDH}, \mathrm{AF}$ e incluso la MS presentada en Zn0 y Mn0. Casierra-Posada et al. (2012) reportan en espinaca (Spinacia oleraceae) que a mayor concentración de zinc el AF decrecía, mientras que para manganeso el efecto no fue tan marcado.

Respecto al AFE no hubo diferencias significativas entre $\mathrm{Zn} 0$ y $\mathrm{Zn} 2$, siendo a su vez mayores al testigo $(P \leq 0,05)$, indicando que tanto en una carencia como un nivel elevado de zinc, las hojas son más delgadas. Analizando la RAF se observó que $Z n 0$ presentó el mayor valor, mientras que Zn1, Zn2 y testigo presentaron un eficiencia similar en la fijación de $\mathrm{CO}_{2}$ por unidad de superficie fotosintética, a pesar de que la MS haya sido diferente. Por su parte, Göransson (1999) encontró en plantas de abedul (Betula pendula), que el AFE y la RAF fueron menores con tasas bajas de suministro de zinc en comparación con 
tasas altas. En pistacho (Pistacia vera) las anteriores variables incrementan su valor a medida que aumenta el nivel de zinc, incluso bajo condiciones de estrés salino (Tavallai et al., 2009)

\section{Boro}

El tratamiento $\mathrm{B} 0$ presentó menor $\mathrm{NDH}$ y AF, respecto los demás niveles del elemento, mientras que no hubo diferencias significativas en AFE y RAF comparado con el testigo $(P \leq 0,05)$. Esto sugiere que hojas carentes de boro fueron relativamente gruesas, y salvando las proporciones, respondieron con la misma eficiencia en la fijación de $\mathrm{CO}_{2}$ por unidad de superficie fotosintética que B1 y testigo (figura 3). En fríjol mungo (Phaseolus aureus) la deficiencia de boro genera reducción tanto en AF como en el AFE, aspecto que indica una mayor densidad celular en hojas debido a la limitada expansión celular (Jiao et al., 2003). El NDH y AF en B1 y B2 fueron significativamente menores al testigo $(P \leq 0,05)$, es decir que niveles bajos y altos limitan estos parámetros. Kaur et al. (2006) encuentran que el exceso de boro reduce el AF en un genotipo de canola (Brassica rapa L.). En tabaco (Nicotiana tabacum) una dosis alta favorece el incremento del AF (Khan et al., 2008). Respecto al AFE, B1 y B2 fueron estadísticamente similares $\mathrm{y}$, al mismo tiempo, mayores que B0 y testigo $(P \leq 0,05)$, mientras que en la RAF no hubo diferencias entre sí, ni con el testigo $(P \leq 0,05)$. Jiao et al. (2003) encuentran en fríjol mungo (Phaseolus aureus) que un nivel tóxico de boro no influencia el AFE, pero sí reduce el AF por abscisión de hojas senescentes, lo que explica en parte el bajo NDH.

\section{Síntomas de deficiencia}

\section{Manganeso}

Debido a la baja movilidad del manganeso en la planta (Broadley et al., 2012) su carencia se evidenció inicialmente en hojas jóvenes, con clorosis generalizada (figura 4A) que afectó los nuevos brotes de la planta (figura 4B), generando abscisión de hojas temprana y abundante. En hojas maduras se observó una clorosis intervenal muy marcada, en donde las nervaduras mantuvieron un color verde oscuro (figura 4C) también aparecieron zonas necróticas rodeadas de halos cloróticas (figura 4D), síntomas similares fueron reportados en maracuyá (Passiflora edulis f. flavicarpa) por Malavolta (1994).

\section{Zinc}

Las plantas que crecieron en condiciones limitadas de $\mathrm{Zn}$ presentaron marcada restricción sobre el tamaño de las hojas (figura 5A). En hojas adultas se desarrolló clorosis desde la margen de los extremos apicales hacia el interior (figura 5B) con evidencia de necrosis en el tejido del ápice (figura 5C). Sintomatología similar ha sido descrita en granadilla (Passiflora ligularis) por Rivera et al. (2002).

\section{Boro}

En general, la carencia de boro correspondió a hojas más pequeñas y gruesas. También se presentó clorosis en hojas jóvenes, que inició en el ápice de los lóbulos (figura 6A), y avanzó hacia la base del limbo con necrosis y entorchamiento sobre su eje, lo que generaba defoliación temprana (figura 6B). En algunas hojas jóvenes se encontró un moteado clorótico generalizado (figura 6C). Los síntomas descritos en hojas concuerdan con lo reportado por García (2002) en plantas de maracuyá.

\section{CONCLUSIONES}

En plantas de curuba en etapa vegetativa fue evidente una restricción en la materia seca en todos los niveles de cada elemento, siendo el nivel de carencia el más limitante. La tendencia en la distribución de masa seca demostró un aumento entre 1 y $14 \%$ de acumulación en raíz para todos los tratamientos en comparación con el testigo. 
En los parámetros foliares, el número de hojas y área foliar fueron limitados en el nivel de carencia de todos los elementos, y en menor proporción los niveles 1 y 2 , con respecto al testigo, lo que explica en parte la baja acumulación de materia seca obtenida. El área foliar específica en todos los tratamientos exceptuando $\mathrm{Mn0}$ y B0 fue alta respecto al testigo, y en la relación de área foliar todos los tratamien- tos exceptuando Mn2 y Zn0 fueron similares al testigo, lo que indica que la tendencia en los diferentes niveles de cada elemento fue a presentar hojas delgadas pero con una producción neta de biomasa por unidad de área foliar similar a la del testigo.

De los tratamientos evaluados, la dosis correspondiente al tratamiento testigo para los ele-
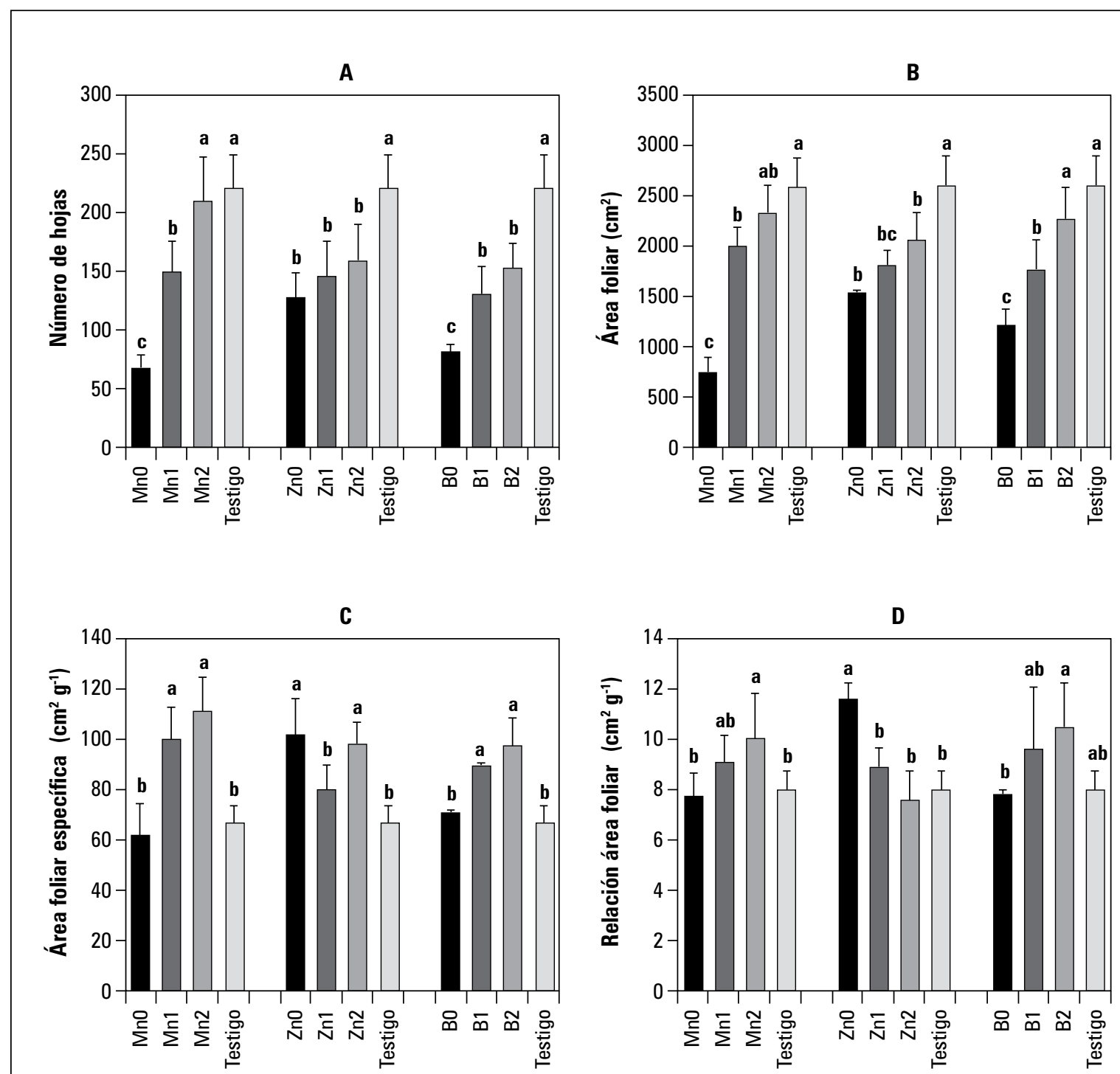

Figura 3. Efecto de diferentes niveles de Mn, Zn y B en: A. Número de hojas (NDH), B. Área foliar (AF), C. Área foliar específica (AFE) y D. Relación área foliar (RAF) en plantas de curuba (Passiflora tripartita var. mollissima). Promedios con letras distintas, entre niveles de un mismo elemento, indican diferencia significativa según la prueba de Duncan $(P \leq 0,05)$. Las barras sobre las columnas indican desviación estándar. 


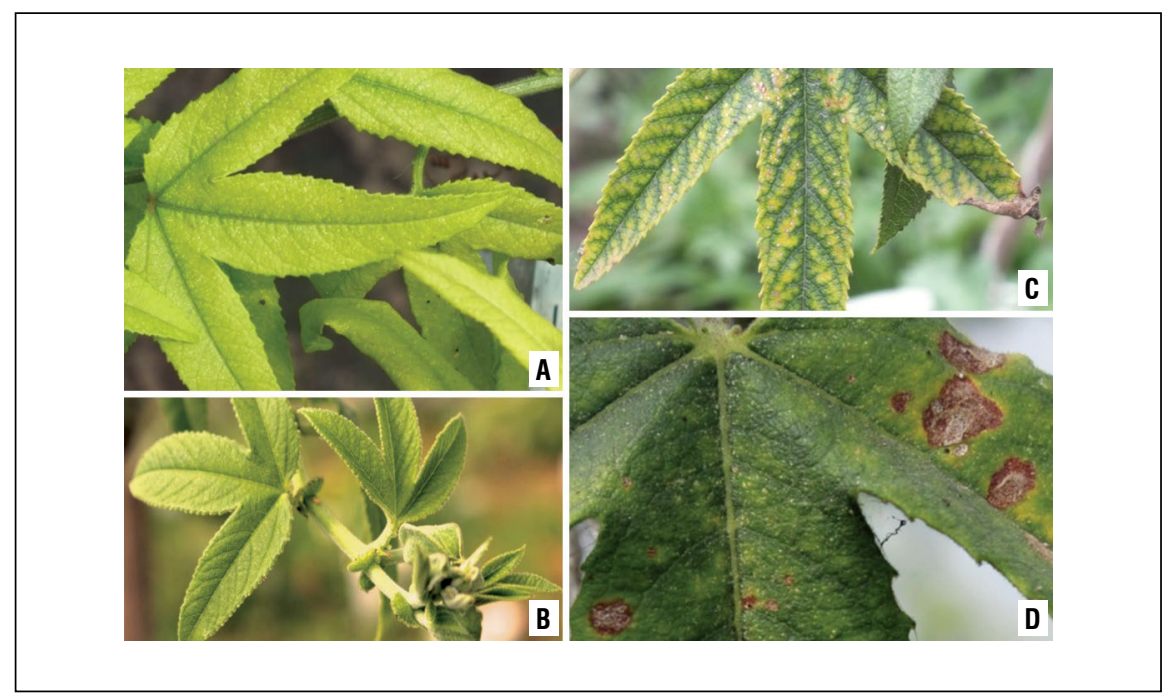

Figura 4. Síntomas de deficiencia de manganeso en hojas de plantas de curuba (Passiflora tripartita var. mollissima): A. Clorosis generalizada en hojas jóvenes, B. Clorosis generalizada en brotes nuevos, C. Clorosis intervenal en hojas maduras y D. Manchas necróticas en hojas maduras. Fotos: 4A, 4C: S. Hernández; 4B, 4D: G. Sánchez.
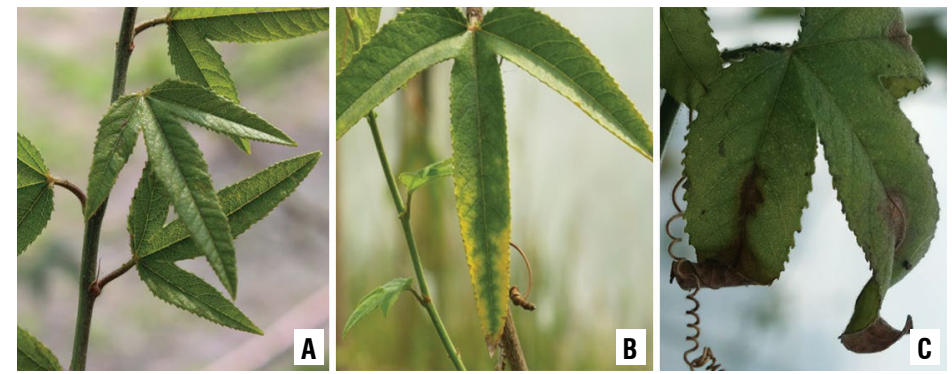

Figura 5. Síntomas de deficiencia de zinc en hojas de plantas de curuba (Passiflora tripartita var. mollissima): A. Lámina foliar reducida, B. Clorosis marginal avanzando hacia el interior en hojas adultas, C. Necrosis de tejido apical en hojas adultas. Fotos: 5B-5C: S. Hernández; 5A: G. Sánchez.

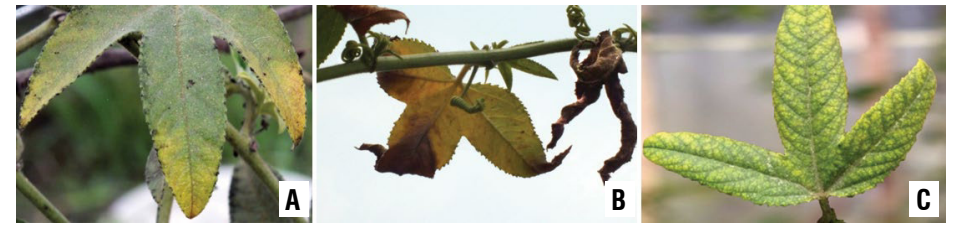

Figura 6. Síntomas de deficiencia de boro en hojas de plantas de curuba (Passiflora tripartita var. mollissima): A. Clorosis apical en lóbulos de hojas adultas, B. Avance de necrosis y enrollamiento en hojas adultas y C. Moteado clorótico generalizado en hojas jóvenes. Fotos: S. Hernández. 
mentos Mn, Zn y B, fue la que presentó los valores más altos en los parámetros evaluados, salvo en AFE y RAF.

La expresión de signos visuales de deficiencia nutricional en hojas de curuba solo ocurrieron en los tratamientos $\mathrm{Mn} 0, \mathrm{Zn} 0$ y B0, la sintomatología clásica para cada elemento. Principalmente con limitación del crecimiento, necrosis, clorosis en distintos patrones, entre otros, lo cual corresponde con lo reportado en otras especies del mismo género.

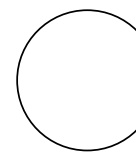

Asad, A., F. Blamey y D. Edwards. 2002. Dry matter production and boron concentrations of vegetative and reproductive tissues of canola and sunflower plants grown in nutrient solution. Plant Soil 243, 243-252.

Broadley, M., P. Brown, I. Cakmak, Z. Rengel y F. Zhao. 2012. Capítulo 7: Function of nutrients: Micronutrients. pp. 191-243. En: Marschner, P. (ed.). Marschner's mineral plant nutrition of higher plants. $3 \mathrm{a}$ ed. Elsevier, Londres.

Cabezas, M. y C. Sánchez. 2008. Efecto de las deficiencias nutricionales en la distribución de la materia seca en plantas de vivero de curuba (Passiflora moIlissima Bailey). Agron. Colomb. 26(2), 197-204.

Cakmak, I., N. Sari, H. Marschner, M. Kalayci, A. Yilmaz, S. Eker y K. Gülüt. 1996. Dry matter production and distribution of zinc in bread and durum wheat genotypes differing in zinc efficiency. Plant Soil 180, 173-181.

Campos, T. 2001. La curuba. Su cultivo. IICA, Bogotá.

Campos, T. y O.C. Quintero. 2012. Curuba (Passiflora tripartita var. mollissima). pp. 421-442. En: Fischer, G. (ed.). Manual para el cultivo de frutales en el trópico. Produmedios, Bogotá.

Casierra-Posada, C. Ulrich y C. Pérez. 2012. Growth of spinach plants (Spinacia oleracea L.) exposed to excess zinc and manganese. Agron. Colomb. 30(3), 344-350.

Casierra-Posada, F. y J. Poveda. 2005. La toxicidad por exceso de Mn y Zn disminuye la producción de materia seca, los pigmentos foliares y la calidad del fruto en fresa (Fragaria sp. cv. Camarosa). Agron. Colomb. 23(2), 283-289.

Casierra-Posada, F., L. González y C. Ulrich. 2010. Crecimiento de plantas de brócoli (Brassica oleracea L. var. Itálica) afectadas por exceso de zinc. Rev. Colomb. Cienc. Hortic. 4(2), 163-174.
REFERENCIAS BIBLIOGRÁFICAS

Cramer, G.R. y R. Nowak. 1992. Supplemental manganese improves the relative growth, net assimilation and photosynthetic rates of salt-stressed barley. Physiol. Plant. 84, 800-805.

Da Silva, D., L. Lanzoni, A. Enedi, L. De Lima y T. Muraoka. 2008. Boron affects the growth and ultraestructure of Castor Been plants. Sci. Agric. 65(6), 659-664.

Feng, Y.L., Y.B. Lei y Z. Li. 2012. Micronutrient deficiencies accelerate leaf senescence in Amomum villosum. Bot. Stud. 53, 345-352.

García, M.A. 2002. Guía técnica. Cultivo de maracuyá amarillo. Centro Nacional de Tecnología Agropecuaria y Forestal, San Salvador.

Goldbach, H., Q. Yu, R. Wingender, M. Schulz, M. Wimmer, P. Findeklee y F. Baluška. 2001. Rapid response reactions of roots to boron deprivation. J. Plant Nutr. Soil Sci. 164, 173-181.

Gómez, M.I. 2012. Fertilización de frutales. pp. 141-168. En: Fischer, G. (ed.). Manual para el cultivo de frutales en el trópico. Produmedios, Bogotá.

González, A. y J.P. Lynch. 1997. Effects of manganese toxicity on leaf $\mathrm{CO}$ assimilation of contrasting common bean genotypes. Physiol. Plant. 101(4), 872-880.

Göransson, A. 1999. Growth and nutrition of Betula pendula at different relative supply rates of zinc. Tree Physiol. 19, 111-116.

Göransson, A. 1994. Growth and nutrition in small Betula pendula plants a different relative addition rates of manganese. Tree Physiol. 14(4), 375-388.

Gupta, U.C. 2007. Boron. pp. 241-277. En: Barker, A. y D. Pilbeam (eds.). Handbook of plant nutrition. Taylor \& Francis Group, Boca Raton, FL. 
Herrera, M., A. González, J. Rexach, J. Camacho, J. Maldonado y M. Navarro. 2010. Role of boron in vascular plants and response mechanisms to boron stresses. Plant Stress 4(2), 115-122.

Hirose, T. 1987. A vegetative plant growth model: Adaptive significance of phenotypic plasticity in matter partitioning. Funct. Ecol. 1(3), 195-202.

Humpries, J.L., J. Stangoulis y R. Graham. 2007. Manganese. pp. 351-374. En: Barker, A. y D. Pilbeam (eds.). Handbook of plant nutrition. Taylor \& Francis Group, Boca Raton, FL.

Hunt, R. 1990. Basic growth analysis. Unwin Hyman, London.

Jiao, X.Y., G. Wang, B. Cheng, H.T. Wang, W.P. Quick y B.C. Jarvis. 2003. Effects of different boron concentrations on the morphology and foliar generalities of mung bean. Acta Ecol. Sin. 23(3), 456-462.

Kaur, S., M.E. Nicolas, R. Ford, R.M. Norton y P.W. Taylor. 2006. Selection of Brassica rapa genotypes for tolerance to boron toxicity. Plant Soil 285, 115-123.

Khan, Z., H. Gul, H. Akber, K. Khan, M. Ikramullah y F. Sha. 2008. Yield and quality of flue-cured Virginia tobacco, Nicotiana tobacum L. as affected by different levels of fico-micron and boron. Sarhad J. Agric. 24(2), 211-216.

Kirkby, E. y V. Römheld. 2008. Micronutrientes en la fisiología de las plantas: Funciones, absorción y movilidad. International Plant Nutrition Institute: Informaciones Agronómicas 68, 1-13.

Kitao, M., T. Lei y T. Kioke. 1999. Effects of manganese in solution culture on the growth of five deciduous broad-leaved tree species with different successional characters from northern Japan. Photosynth. 36(1-2), 31-40.

László, M. 2008. Manganese requirement of sunflower (Helianthus annuus L.), tobacco (Nicotiana tabacum L.) and triticale ( $x$ Triticosecale W.) at early stage of growth. Europ. J. Agron. 28, 586-596.

Lei, Y., H. Korpelainen y C. Li. 2007. Physiological and biochemical responses to high $\mathrm{Mn}$ concentrations in two contrasting Populus cathayana populations. Chemosphere 68, 686-694.

Lizarazo, M.A., C.A. Hernández, G. Fischer y M.I. Gómez. 2013. Biomass, foliar parameters and symptomatology in response to different levels of nitrogen, potassium and magnesium in banana passion fruit (Passiflora tripartita var. mollissima). Agron. Colomb. 31(2) (en imprenta).

Loomis, W.D. y R.W. Durst. 1992. Chemistry and biology of boron. BioFactors 3, 229-239.
Lukaszewski, K.M. y D. Blevins. 1996. Root growth inhibition in boron-deficient or aluminum-stressed squash may be a result of impaired ascorbate metabolism. Plant Physiol. 112, 1135-1140.

Malavolta, E. 1994. Nutrición y fertilización del maracuyá. International Plant Nutrition Institute, Quito.

Marschner, H., E.A. Kirkby e I. Cakmak. 1996. Effect of mineral nutritional status on shoot-root partitioning of photoassimilates and cycling of mineral nutrients. J. Exp. Bot. 47, 1255-1263.

McCarthy, M.C. y B.J. Enquist. 2007. Consistency between an allometric approach and optimal partitioning theory in global patterns of plant biomass allocation. Funct. Ecol. 21(4), 713-720.

Millaleo, R., M. Reyes-Díaz, A.G. Ivanov, M.L. Mora y M. Alberdi. 2010. Manganese as essential and toxi element for plants: Transport, accumulation and resistance mechanisms. J. Soil Sci. Plant Nutr. 10(4), 476-494.

Miranda, D. y C. Carranza. 2010. Caracterización de sistemas productivos de pasifloráceas en Colombia. pp. 27-59. En: Memorias I Congreso Latinoamericano de Pasiflora. Corporación Cepass Huila y Asohofrucol, Neiva, Colombia.

Mou, D., Y. Yaoa, Y. Yang, Y. Zhang, C. Tiana y V. Achal. 2011. Plant high tolerance to excess manganese related with root growth, manganese distribution and antioxidative enzyme activity in three grape cultivars. Ecotoxicol. Environ. Safety. 74, 776-786.

Ohki, K. 1984. Manganese deficiency and toxicity effects on growth, development and nutrient composition in wheat. Agron. J. 76, 213-218.

Primot, S., V. Rioux, G.C. d`Eeckenbrugge, F. Garcin y J.A. Ocampo. 2005. Variación morfológica de tres especies de curubas (Passiflora tripartita var. mollissima, P. tarminiana y P. mixta) y sus híbridos en el Valle del Cauca (Colombia). Rev. Bras. Frutic. 27(3), 467-471.

Rivera, B., D. Miranda, L.A. Ávila y A.M. Nieto. 2002. Manejo integral del cultivo de la granadilla (Passiflora ligularis Juss.). Editorial Litoas, Manizales, Colombia.

Santiago, L., S. Wright, K. Harms, J. Yavitt, C. Korine, M. García y B. Turner. 2012. Tropical tree seedling growth responses to nitrogen, phosphorus and potassium addition. J. Ecol. 100(2), 309-316.

Singh, P. A. Misra y N.K. Srivastava. 2001. Influence of $\mathrm{Mn}$ deficiency on growth, chlorophyll content, 
physiology and essential monoterpene oil(s) in genotypes of spearmint (Mentha spicata L.). Photosynthetica 39(3), 473-476.

Tavallali, V., M. Rahemi y B. Kholdebarin. 2009. Ameliorative effects of zinc on pistachio (Pistacia vera $\mathrm{L}$.) growth under salt-affected soil conditions. Res. J. Environ. Sci. 3(6), 656-666.

Thompson, L.M. y F. Troeh. 1988. Los suelos y su fertilidad. $4^{a}$ ed. Editorial Reverté S.A., Barcelona, España.

Weiland, R.T., R. Noble y R. Crang. 1975. Photosynthetic and chloroplast ultrastructural consequences of manganese deficiency in soybean. Amer. J. Bot. 62(5), 501-508.

Wilkinson, R. y K. Ohki. 1988. Influence of manganese deficiency and toxicity on isoprenoid synthesis. Plant Physiol. 87, 841-846.

Wilson, J.B. 1988. A review of evidence on the control of shoot:root ratio, in relation to models. Ann. Bot. 61, 443-449.

Zahoor, R., S. Ahmad, H. Munir, M. Ashfaq y S. Yousaf. 2011. Role of boron in improving assimilate partitioning and achene yield in sunflower. J. Agri. Soci. Sci. 7(2), 49-55. 